\title{
Improved Results on Robust Stability Analysis and Stabilization for a Class of Uncertain Nonlinear Systems
}

\section{Mohamed Moez Belhaouane, Mohamed Faiez Ghariani, Hela Belkhiria Ayadi, and Naceur Benhadj Braiek}

\author{
Laboratoire d'Etude et Commande Automatique de Processus (LECAP), \\ Ecole Polytechnique de Tunisie, BP 743, 2078 La Marsa, Tunisia \\ Correspondence should be addressed to Mohamed Moez Belhaouane, \\ moez.belhaouane@ept.rnu.tn
}

Received 24 April 2010; Accepted 3 November 2010

Academic Editor: Jerzy Warminski

Copyright (c) 2010 Mohamed Moez Belhaouane et al. This is an open access article distributed under the Creative Commons Attribution License, which permits unrestricted use, distribution, and reproduction in any medium, provided the original work is properly cited.

\begin{abstract}
This paper deals with the problems of robust stability analysis and robust stabilization for uncertain nonlinear polynomial systems. The combination of a polynomial system stability criterion with an improved robustness measure of uncertain linear systems has allowed the formulation of a new criterion for robustness bound estimation of the studied uncertain polynomial systems. Indeed, the formulated approach is extended to involve the global stabilization of nonlinear polynomial systems with maximization of the stability robustness bound. The proposed method is helpful to improve the existing techniques used in the analysis and control for uncertain polynomial systems. Simulation examples illustrate the potentials of the proposed approach.
\end{abstract}

\section{Introduction}

Being subject of considerable theoretical and practical significance, stability analysis and control of nonlinear dynamic systems have been attracting the interest of investigators for several decades [1-3]. The essential aim of robust analysis and nonlinear robust control theory is to internally stabilize the nonlinear plant while maximizing the upper bound on the parametric perturbations, such that the perturbed nonlinear system remains stable, as described in [4-8]. However, each of the published approaches on this subject concerns particular classes of nonlinear uncertain systems and there is no standard method to investigate robust stability and stabilization of general high-order nonlinear systems [913]. Therefore, in deep contrast with linear analysis and control methods, which are flexible, 
efficient and allowing to solve a broad class of linear control problems, there are few practical methods in nonlinear control which can handle real engineering problems with similar comfort.

In this paper, we are concerned with further developments of robust stability and feedback stabilization methods of a class of nonlinear polynomial systems with structured uncertainties. Our motivations for studying polynomial systems mainly comes from the fact that they provide a convenient unified framework for mathematical modelling of many physical processes and practical applications such as electrical machines and robot manipulators, and also the ability to approach any analytical nonlinear dynamical systems, since, any nonlinear system can be developed into a polynomial form by Taylor series expansions [14-17]. Moreover, the description of polynomial systems can be simplified using the Kronecker product and power of vectors and matrices [18]. The parameter uncertainties in the studied polynomial systems are considered as real structured perturbations which are assumed to depend linearly on a set of interval parameters.

The purpose of this work is then to find a bound on the size of the parameters for which the perturbed model remains stable as well as design robustly state feedback controller for the above-mentioned systems. The presented research is built on the stability and stabilization conditions of nonlinear polynomial systems proposed in $[16,19,20]$ and the measure of stability robustness of linear systems [12], and makes use of a duality principle between the linear and nonlinear uncertain systems [21, 22]. The derived results are formulated in terms of nonlinear optimization problem which is easy to testify by using Matlab Tool-Box. Let's mention that previous works of the same research team [21, 22] had considered the robustness measure of polynomial systems using the Yedavalli robustness measure of linear uncertain systems [10]. In the present contribution we aim to improve these previous results by considering a less conservative robustness criterion and to apply the obtained bound formulation to the robust control synthesis.

This article is organized as follows. Section 2 contains the mathematical notations and preliminary material on algebraic properties of the Kronecker product and power of vectors and matrices. Section 3 formulates the problem and the description of the nonlinear studied systems. Sections 4 and 5 present our main results, which concern the development of the stability robustness measurement and the design of a robust polynomial state feedback controller with robustness maximization. Numerical examples illustrating the effectiveness of the proposed methods are provided. Finally, a conclusion ends the paper.

\section{Mathematical Notations and Useful Tools}

We use standard notations throughout this paper. $\mathbb{R}^{n}$ denotes the $n$-dimensional Euclidean space, $\mathbb{R}^{n \times m}$ is the set of all real $m \times n$ matrices, and $\otimes$ is the symbol of the Kronecker product. $0_{n \times m}:(n \times m)$ zero matrix, 0 : zero matrix of convenient dimension, $A^{T}$ is the transpose of matrix $A, A>0(A \geq 0)$ is the symmetric positive definite (semidefinite) matrix. The $k$ th row of a matrix such as $A$ is denoted $A^{k}$ and the $i k$ element of $A$ will be denoted $a_{i k}$. The identity matrix of order $m$ is denoted as $I_{m}$ (or simply $I$ if no confusion arises). $|\cdot|$ stands for the matrix formed by taking the componentwise absolute value of the matrix. $\lambda(\cdot)$ is the eigenvalue of the considered matrix. $\rho(\cdot)=\left|\lambda_{\max }(\cdot)\right|$ is the spectral radius of the indicated matrix.

In this section, we propose some algebraic tools and definitions needed to demonstrate the robustness measure and the stabilizing control, of the studied systems, in the next sections. 


\subsection{Kronecker's Power of Vectors}

The Kronecker power of order $i$, denoted $X^{[i]}$, of the vector $X \in \mathbb{R}^{n}$ is defined as (see [18]):

$$
\begin{gathered}
X^{[0]}=1, \\
X^{[i]}=X^{[i-1]} \otimes X=X \otimes X^{[i-1]} \quad \text { for } i \geq 1 .
\end{gathered}
$$

For example, considering the second order, the $X^{[2]}$ vector is given by

$$
X^{[2]}=\left[x_{1}^{2}, x_{1} x_{2}, \ldots, x_{1} x_{n}, x_{2} x_{1}, \ldots, x_{2} x_{n}, \ldots, x_{n} x_{1}, \ldots, x_{n}^{2}\right]^{T},
$$

$\tilde{X}_{i=1, \ldots, r}^{[i]} \in \mathbb{R}^{n_{i}}, n_{i}=\left(\begin{array}{c}n+i-1 \\ i\end{array}\right)$ is the nonredundant Kronecker power of the state vector $X$ defined as

$$
\begin{gathered}
\tilde{X}^{[1]}=X^{[1]}=X, \\
\forall i \geq 2, \quad \tilde{X}^{[i]}=\left[x_{1}^{i}, x_{1}^{i-1} x_{2}, \ldots, x_{1}^{i-1} x_{n}, \ldots, x_{1}^{i-2} x_{n}^{2}, \ldots, x_{1}^{i-3} x_{2}^{3}, \ldots, x_{n}^{i}\right]^{T},
\end{gathered}
$$

where the repeated components of the redundant $i$ th-power $X^{[i]}$ are omitted.

The relation between the redundant and the nun-redundant Kronecker power of the vector $X$ can be stated as follows:

$$
\begin{gathered}
\forall i \in \mathbb{N} \exists ! \quad T_{i} \in \mathbb{R}^{n^{i} \times n_{i}}, \\
X^{[i]}=T_{i} \tilde{X}^{[i]},
\end{gathered}
$$

where $T_{i}$ is called the transition matrix which a procedure of the determination is given in [23].

\subsection{Mat-Function: mat(·)}

If $V$ is a vector of dimension $p=n \cdot m$, then $M=\operatorname{mat}_{(n, m)}(V)$ is the $(n \times m)$ matrix verifying $V=\operatorname{vec}(M)$.

\subsection{Vec-Function: $\operatorname{vec}(\cdot)$}

An important vector valued function of matrix denoted vec $(\cdot)$ was defined in [18] as follows:

$$
\mathcal{C}=\left[\begin{array}{llll}
c_{1} & c_{2} & \cdots & c_{q}
\end{array}\right] \in \mathbb{R}^{p \times q},
$$

where for all $i \in\{1, \ldots, q\}, c_{i} \in \mathbb{R}^{p}$ are the columns of $\mathcal{C}$

$$
\operatorname{vec}(\mathcal{C})=\left[\begin{array}{llll}
c_{1}^{T} & c_{2}^{T} & \cdots & c_{q}^{T}
\end{array}\right]^{T} \in \mathbb{R}^{p q} .
$$




\subsection{Polynomial Vectorial Function}

For a polynomial vectorial function denoted $a(X)$ given as follows:

$$
a(X)=\sum_{i=1}^{r} A_{i} X^{[i]}
$$

where $X \in \mathbb{R}^{n}, A_{i}$ are $\left(n \times n^{i}\right)$ constant matrices and $r$ is the polynomial order (considered odd) defined by $r=2 s-1$, with $s \in \mathbb{N}^{*}$.

We define the $(v \times v)$ matrix $\mathcal{M}(a)$ as

$$
M(a)=\left[\begin{array}{ccccc}
M_{11}\left(A_{1}\right) & M_{12}\left(A_{2}\right) & 0 & \ldots & 0 \\
0 & M_{22}\left(A_{3}\right) & \ddots & \ddots & \vdots \\
\vdots & \ddots & \ddots & \ddots & 0 \\
\vdots & & \ddots & M_{s-1, s-1}\left(A_{2 s-3}\right) & M_{s-1, s}\left(A_{2 s-2}\right) \\
0 & \ldots & \ldots & 0 & M_{s, s}\left(A_{2 s-1}\right)
\end{array}\right]
$$

where $v=n+n^{2}+\cdots+n^{s}$, and

(i) for $j=1, \ldots, s$

$$
M_{j, j}\left(A_{2 j-1}\right)=\left[\begin{array}{c}
\operatorname{mat}_{\left(n^{j-1}, n^{j}\right)}\left(A_{2 j-1}^{1 T}\right) \\
\operatorname{mat}_{\left(n^{j-1}, n^{j}\right)}\left(A_{2 j-1}^{2 T}\right) \\
\vdots \\
\operatorname{mat}_{\left(n^{j-1}, n^{j}\right)}\left(A_{2 j-1}^{n T}\right)
\end{array}\right],
$$

(ii) for $j=1, \ldots, s-1$

$$
\begin{aligned}
& M_{j, j+1}\left(A_{2 j}\right)=\left[\begin{array}{c}
\operatorname{mat}_{\left(n^{j-1}, n^{j}\right)}\left(A_{2 j}^{1 T}\right) \\
\operatorname{mat}_{\left(n^{j-1}, n^{j}\right)}\left(A_{2 j}^{2 T}\right) \\
\vdots \\
\operatorname{mat}_{\left(n^{j-1}, n^{j}\right)}\left(A_{2 j}^{n T}\right)
\end{array}\right], \\
& A_{k}=\left[\begin{array}{llll}
A_{k}^{1 T} & A_{k}^{2} & \cdots & A_{k}^{n T}
\end{array}\right] .
\end{aligned}
$$




\section{System Description and Problem Formulation}

Consider the class of uncertain nonlinear polynomial systems with structured uncertainties described by

$$
\dot{X}=f(X)+\Delta f(X)+B U,
$$

where

$$
\begin{gathered}
f(X)=\sum_{k=1}^{r} F_{k} X^{\{k\}}, \\
\Delta f(X)=\sum_{k=1}^{r} \Delta F_{k} X^{[k]},
\end{gathered}
$$

with

(i) $X=\left[x_{1}, \ldots, x_{n}\right]^{T} \in \mathbb{R}^{n}$ is the state vector,

(ii) $X^{[k]} \in \mathbb{R}^{n^{k}}$ is the $k$ th Kronecker power of the vector $X$,

(iii) $U \in \mathbb{R}^{m}$ is the control input vector,

(iv) $F_{k} \in \mathbb{R}^{n \times n^{k}}$ are constant matrices which describe the nominal system,

(v) $B \in \mathbb{R}^{n \times m}$ is the constant control matrix,

(vi) $\Delta F_{k, k=1, \ldots, r} \in \mathbb{R}^{n \times n^{k}}$ are the matrices of parametric uncertainties.

We note that the structure of the parametric uncertainties $\Delta F_{k, k=1, \ldots, r}$ is multipleparameter variations given by

$$
\Delta F_{k}=\sum_{j=1}^{\gamma_{k}} \varepsilon_{k j} E_{k j}
$$

where $E_{k j} \in \mathbb{R}^{n \times n^{k}}$ (for $k=1, \ldots, r$ and $j=1, \ldots, \gamma_{k}$ ) are numerically known matrices, $\varepsilon_{k j}$ represent the uncertain parameters, and $\gamma_{k}$ represents the number of uncertain parameters of the matrix $F_{k}$.

According to (3.1) and (3.3), let $F_{k, k=1, \ldots, r}$ be some nominal matrices which present the parameters of the considered polynomial system. Define the perturbed terms which depend linearly on a number of parameters $\varepsilon_{k j}$ which are unknown real scalars.

The objective of this paper is to address the following problems:

(1) in the robust stability analysis, it is assumed that the nominally system described by

$$
\dot{X}=f(X)=\sum_{k=1}^{r} F_{k} X^{[k]}
$$


is globally asymptotically stable, and we wish to find an upper bound $\eta$ of the parameters $\varepsilon_{k j}\left(\max \left(\varepsilon_{k j}\right) \leq \eta\right)$ such that the following nonlinear polynomial systems without controller (for $U(t)=0$ ):

$$
\dot{X}=f(X)+\Delta f(X)=\sum_{k=1}^{r}\left(F_{k}+\Delta F_{k}\right) X^{[k]}
$$

remains stable, and

(2) the robust control problem consist to design a nonlinear robust controller which ensures the global stability of the nominal nonlinear systems (3.4) and maximizes the stability robustness of the controlled system. This means that the stability of the controlled system is ensured for a maximum value of the uncertainties affecting the system parameters.

In the next sections, we will address the robustness analysis and synthesis problems for nonlinear polynomial systems with the above described admissible structured uncertainty set.

\section{Robustness Measure}

This section is devoted to the development of a robustness stability measure of the above class of nonlinear systems. Our main result in this work is based on the duality principle, presented in the sequel, between the computation of stability robustness bound approach of linear systems and a previous result about the stability analysis of certain polynomial systems. First, we beginning by presenting the following basic results.

\subsection{Stability Robustness Bound for Linear System}

The problem of robust stability of linear state-space systems, for both structured and unstructured parametric uncertainty involving state space models, has been an active area of research for quite some time for extensive discussions and references. Let the following linear dynamical system with linear structured perturbations:

$$
\dot{X}=A X(t)+\left(\sum_{k=1}^{p} \varepsilon_{k} E_{k}\right) X(t)=(A+\Delta A) X(t),
$$

where

$$
\Delta A=\sum_{k=1}^{p} \varepsilon_{k} E_{k}
$$

with $A \in \mathbb{R}^{n \times n}$ is a nominal Hurwitz matrix with eigenvalues in the open left-half plane, $\Delta A$ presents the additive uncertainty with $E_{k} \in \mathbb{R}^{n \times n}$ are constant known matrices and $\varepsilon_{k, k=1, \ldots, p}$ denote the unknown real parameters. Note that the uncertain parameters enter the uncertainty matrix linearly. 
As mentioned in the first section, different results have been obtained for determining the extent of uncertainty that the system can tolerate without becoming unstable. However, it remains challenging to develop methods for finding less conservative robustness bounds in the presence of structured perturbations. we present here two results which have been applied in our work.

\subsubsection{Robustness Measure of Yedavalli}

Consider an uncertain linear system described by (4.1) with structured uncertainties given by (4.2), then it comes out the following Yedavalli result [10].

Theorem 4.1. The uncertain linear system (4.1)-(4.2) is asymptotically stable if:

$$
\max \left|\varepsilon_{k}\right| \leq\left(\rho\left\{\sum_{k=1}^{p}\left|\Gamma_{k} \Phi^{-1}\right|\right\}\right)^{-1}
$$

with

$$
\begin{gathered}
\Gamma_{k}=E_{k} \otimes I_{n}+I_{n} \otimes E_{k} \\
\Phi=A \otimes I_{n}+I_{n} \otimes A .
\end{gathered}
$$

\subsubsection{Robustness Measure of Gardiner}

Next, we review an improved robustness algorithm proposed by Gardiner that gives a better bound than the methods obtained in [9] and Yedavalli's works [10, 24, 25] presented above. Therefore, the following theorem provides am improved robustness bound of linear uncertain system [12].

Theorem 4.2. The system (4.1)-(4.2) is asymptotically stable if

$$
\max \left|\varepsilon_{k}\right| \leq\left(\rho\left\{\sum_{k_{1}=1}^{p} \sum_{k_{2}=1}^{k_{1}-1}\left|\Xi_{k_{1}} A_{0}^{-1} \Xi_{k_{2}} A_{0}^{-1}+\Xi_{k_{2}} A_{0}^{-1} \Xi_{k_{1}} A_{0}^{-1}\right|+\sum_{k=1}^{p}\left|\left(\Xi_{k} A_{0}^{-1}\right)^{2}\right|\right\}\right)^{-1 / 2},
$$

with

$$
\begin{gathered}
\Xi_{k}=E_{k} \otimes I_{n}+I_{n} \otimes E_{k}, \\
\Xi_{k_{i}}=E_{k_{i}} \otimes I_{n}+I_{n} \otimes E_{k_{i}}, \\
A_{0}=A \otimes I_{n}+I_{n} \otimes A .
\end{gathered}
$$

Before applying these two results for general high-order nonlinear polynomial systems, we assess recent algebraic stability criterion for the polynomial systems in the certain case. 


\subsection{Stability Criterion of Nonlinear Polynomial Systems}

The robustness measure developed in this study is specially based on the pertinent results presented in the following theorem [15, 16, 19], about the global stability condition of autonomous polynomial systems described by (3.4). This stability condition is based on the Lyapunov direct method with quadratic Lyapunov function $\mho(X)=X^{T} P X\left(P \in \mathbb{R}^{n \times n}\right)$ [26].

Theorem 4.3. The nonlinear polynomial system defined by (3.4) is globally asymptotically stable if there exist:

(i) an $(n \times n)$-symmetric positive definite matrix $P$, and

(ii) arbitrary parameters $\mu_{i, i=1, \ldots, \beta} \in \mathbb{R}$,

such that the $(\vartheta \times \vartheta)$ symmetric matrix $Q$ defined by

$$
Q=\varphi\left(P, \mu_{i, i=1, \ldots, \beta}\right)=\tau^{T}\left(p \mathcal{M}(f)+\mathcal{M}(f)^{T} p\right) \tau+\Pi\left(\mu_{i, i=1, \ldots, \beta}\right)
$$

is negative definite.

The proof elements and the parameters notation of Theorem 4.3 are illustrated in appendix (see $[15,16,19,20]$ for more details).

\subsection{Robustness Stability Measure of Uncertain Polynomial Systems}

\subsubsection{Duality Approach}

Refrying to the stability condition of certain polynomial system (4.7), given by Theorem 4.1, the uncertain nonlinear polynomial system (3.5) is globally asymptotically stable, if there exist a positive definite matrix $P$ and real parameters $\mu_{i, i=1, \ldots, \beta}$ such that:

$$
\widetilde{Q}=\mathcal{Q}+\Delta Q=\varphi\left(F_{k}+\Delta F_{k, k=1, \ldots, r}\right)<0,
$$

where

$$
\begin{gathered}
Q=\varphi\left(F_{k, k=1, \ldots, r}\right), \\
\Delta Q=\varphi\left(\Delta F_{k, k=1, \ldots, r}\right) .
\end{gathered}
$$

It can be verified that

$$
\begin{gathered}
\varphi\left(\Delta F_{k, k=1, \ldots, r}\right)=\sum_{k=1}^{r} \sum_{j=1}^{\gamma_{k}} \varepsilon_{k j} \Xi_{k j}, \\
\Xi_{k j}=\varphi\left(\Lambda_{i j, i=1, \ldots, r}\right), \quad \Lambda_{i j}= \begin{cases}E_{k j} & \text { if } i=k, \\
0 & \text { if } i \neq k .\end{cases}
\end{gathered}
$$


Indeed, the system (3.5) is globally asymptotically stable if the matrix $\widetilde{Q}$ is negative definite (i.e., when $\left.\lambda_{i}(\tilde{\mathcal{Q}})<0, i=1, \ldots, \vartheta\right)$. Then, to ensure the negativity of $\tilde{\mathcal{Q}}$, it will be sufficient to guarantee the stability of the following linear uncertain system:

$$
\dot{z}=Q \mathcal{Z}+\left(\sum_{k=1}^{r} \sum_{j=1}^{\gamma_{k}} \varepsilon_{k j} \Xi_{k j}\right) \mathfrak{z}
$$

said linear dual system of nonlinear system (3.5). So, the robustness measure of the nonlinear system (3.5) is equivalent to the robustness measure of the linear dual system (4.11).

In the following we will apply some results on linear robustness measures determination for characterizing a robustness measure of nonlinear polynomial systems. In this way, two results will be presented. A previous result based on the Yedavalli linear robustness measure [21] and a new improved result using the Gardiner linear robustness bound (4.5).

\subsubsection{Yedavalli Based Robustness Measure of Polynomial Systems}

Let consider the uncertain nonlinear polynomial system (3.5), We assume that the nominal system satisfies the stability criterion (4.7) of Theorem 4.3, then we obtain the following result formulated in Theorem 4.4 [21].

Theorem 4.4. The nonlinear uncertain polynomial system (3.5) is robustly stable, if

$$
\eta_{1}=\max \left|\varepsilon_{k j}\right| \leq \eta_{1}^{\star}
$$

with

$$
\eta_{1}^{\star}=\max _{p}\left(\rho\left\{\sum_{k=1}^{r} \sum_{j=1}^{\gamma_{k}}\left|\left(\Omega_{k j} \Theta^{-1}\right)\right|\right\}\right)^{-1}
$$

where for $k=1, \ldots, r, j=1, \ldots, \gamma_{k}$

$$
\begin{gathered}
\Omega_{k j}=\varepsilon_{k j} \otimes I_{\vartheta}+I_{\vartheta} \otimes \varepsilon_{k j}, \\
\Theta=Q \otimes I_{\vartheta}+I_{\vartheta} \otimes \mathcal{Q}, \\
\Xi_{k j}=\varphi\left(\Lambda_{i j, i=1, \ldots, r}\right) ; \quad \Lambda_{i j}= \begin{cases}E_{k j} & \text { if } i=k, \\
0 & \text { if } i \neq k,\end{cases} \\
\vartheta=\sum_{k=1}^{s}\left(\begin{array}{c}
n+k-1 \\
k
\end{array}\right) .
\end{gathered}
$$

This theorem was proved in [21]. 


\subsubsection{Gardiner Based Robustness Measure of Polynomial Systems}

We present now the proposed robustness bound of uncertain polynomial systems based on the Gardiner's method.

Theorem 4.5. The nonlinear uncertain polynomial system (3.5) is robustly stable, if

$$
\eta_{2}=\max \left|\varepsilon_{k j}\right| \leq \eta_{2}^{\star}
$$

with

$$
\eta_{2}^{\star}=\max _{P, \mu_{i, i=1, \ldots, \beta}}\left(\rho\left\{\sum_{k_{1}, j_{1}} \sum_{k_{2}, j_{2}}\left|\Omega_{k_{1} j_{1}} \Theta^{-1} \Omega_{k_{2} j_{2}} \Theta^{-1}+\Omega_{k_{2} j_{2}} \Theta^{-1} \Omega_{k_{1} j_{1}} \Theta^{-1}\right|+\sum_{k, j}\left|\left(\Omega_{k j} \Theta^{-1}\right)^{2}\right|\right\}\right)^{-1 / 2},
$$

where for $k_{1}=1, \ldots, r, k_{2}=1, \ldots, k_{1}-1, j_{1}=1, \ldots, \gamma_{k_{1}}$ and $j_{2}=1, \ldots, \gamma_{k_{2}}$

$$
\begin{gathered}
\Omega_{k j}=\Xi_{k j} \otimes I_{\vartheta}+I_{\vartheta} \otimes \Xi_{k j,} \\
\Omega_{k_{i} j_{i}}=\Xi_{k_{i} j_{i}} \otimes I_{\vartheta}+I_{\vartheta} \otimes \Xi_{k_{i} j_{i}}, \\
\Theta=Q \otimes I_{\vartheta}+I_{\vartheta} \otimes \mathcal{Q}, \\
\Xi_{k j}=\varphi\left(\Lambda_{l j, l=1, \ldots, r}\right) ; \quad \Lambda_{l j}= \begin{cases}E_{k j} & \text { if } l=k, \\
0 & \text { if } l \neq k,\end{cases} \\
\Xi_{k_{i} j_{i}}=\varphi\left(\Lambda_{l j_{i}, l=1, \ldots, r}\right) ; \quad \Lambda_{l j_{i}}= \begin{cases}E_{k_{i} j_{i}} & \text { if } l=k_{i}, \\
0 & \text { if } l \neq k_{i} .\end{cases}
\end{gathered}
$$

Proof. The stability of the polynomial system (3.5) is equivalent to that of the dual linear system (4.11). Then, applying the above Gardiner criterion [12] leads to the result of Theorem 4.5, which completes the proof.

Let us remark that Theorem 4.5 leads an improved robustness bound, since it is based on the linear Gardiner measure which is proved larger than Yedavalli one. Moreover, the optimization of the new measure is made with regard to $P$ matrix and the $\mu_{i}$-parameters while the previous measure given in Theorem 4.4 is optimized only with regard to the Lyapunov matrix $P$. 
Mathematical Problems in Engineering

\subsection{An Illustrative Numerical Example}

Consider the following second-order autonomous uncertain polynomial system:

$$
\begin{aligned}
\dot{x}_{1}= & \left(a_{11}+\Delta a_{11}\right) x_{1}+\left(b_{11}+\Delta b_{11}\right) x_{1}^{2}+\left(b_{13}+\Delta b_{13}\right) x_{2}^{2} \\
& +\left(c_{11}+\Delta c_{11}\right) x_{1}^{3}+\left(c_{14}+\Delta c_{14}\right) x_{2}^{3}, \\
\dot{x}_{2}= & \left(a_{22}+\Delta a_{22}\right) x_{2}+\left(b_{21}+\Delta b_{21}\right) x_{1}^{2}+\left(b_{23}+\Delta b_{23}\right) x_{2}^{2} \\
& +\left(c_{21}+\Delta c_{21}\right) x_{1}^{3}+\left(c_{24}+\Delta c_{24}\right) x_{2}^{3} .
\end{aligned}
$$

This system can be written in the following polynomial form:

$$
\dot{X}=\left(F_{1}+\Delta F_{1}\right) X+\left(F_{2}+\Delta F_{2}\right) X^{[2]}+\left(F_{3}+\Delta F_{3}\right) X^{[3]},
$$

where

$$
\begin{aligned}
& F_{1}=\left[\begin{array}{cc}
a_{11} & 0 \\
0 & a_{22}
\end{array}\right], \quad F_{2}=\left[\begin{array}{llll}
b_{11} & 0 & 0 & b_{13} \\
b_{21} & 0 & 0 & b_{23}
\end{array}\right], \quad F_{3}=\left[\begin{array}{llllllll}
c_{11} & 0 & 0 & 0 & 0 & 0 & 0 & c_{14} \\
c_{21} & 0 & 0 & 0 & 0 & 0 & 0 & c_{24}
\end{array}\right], \\
& \Delta F_{1}=\Delta a_{11}\left[\begin{array}{ll}
1 & 0 \\
0 & 0
\end{array}\right]+\Delta a_{22}\left[\begin{array}{ll}
0 & 0 \\
0 & 1
\end{array}\right]=\Delta a_{11} E_{11}+\Delta a_{22} E_{12} \\
& \Delta F_{2}=\Delta b_{11}\left[\begin{array}{llll}
1 & 0 & 0 & 0 \\
0 & 0 & 0 & 0
\end{array}\right]+\Delta b_{13}\left[\begin{array}{llll}
0 & 0 & 0 & 1 \\
0 & 0 & 0 & 0
\end{array}\right]+\Delta b_{21}\left[\begin{array}{llll}
0 & 0 & 0 & 0 \\
1 & 0 & 0 & 0
\end{array}\right]+\Delta b_{23}\left[\begin{array}{llll}
0 & 0 & 0 & 0 \\
0 & 0 & 0 & 1
\end{array}\right] \\
& =\Delta b_{11} E_{21}+\Delta b_{13} E_{22}+\Delta b_{21} E_{23}+\Delta b_{23} E_{24} \text {, } \\
& \Delta F_{3}=\Delta c_{11}\left[\begin{array}{llllllll}
1 & 0 & 0 & 0 & 0 & 0 & 0 & 0 \\
0 & 0 & 0 & 0 & 0 & 0 & 0 & 0
\end{array}\right]+\Delta c_{14}\left[\begin{array}{llllllll}
0 & 0 & 0 & 0 & 0 & 0 & 0 & 1 \\
0 & 0 & 0 & 0 & 0 & 0 & 0 & 0
\end{array}\right] \\
& +\Delta c_{21}\left[\begin{array}{llllllll}
0 & 0 & 0 & 0 & 0 & 0 & 0 & 0 \\
1 & 0 & 0 & 0 & 0 & 0 & 0 & 0
\end{array}\right]+\Delta c_{24}\left[\begin{array}{llllllll}
0 & 0 & 0 & 0 & 0 & 0 & 0 & 0 \\
0 & 0 & 0 & 0 & 0 & 0 & 0 & 1
\end{array}\right] \\
& =\Delta c_{11} E_{31}+\Delta c_{14} E_{32}+\Delta c_{21} E_{33}+\Delta c_{24} E_{34},
\end{aligned}
$$

with:

$$
\begin{gathered}
a_{11}=-0.839, \quad a_{22}=1.921, \quad b_{11}=-0.751, \\
b_{13}=-0.432, \quad b_{21}=-1.673, \\
b_{23}=-2.786, \quad c_{11}=-0.753, \quad c_{14}=-0.634, \\
c_{21}=-0.532, \quad c_{24}=-2.431 .
\end{gathered}
$$


Then, we have the following uncertain parameters:

$$
\left[\varepsilon_{i j}\right]=\left[\begin{array}{rl}
\Delta a_{i j}, & i=1,2 ; j=1,2 \\
\Delta b_{i j}, & i=1,2 ; j=1,3 \\
\Delta c_{i j}, & i=1,2 ; j=1,4
\end{array}\right]
$$

Using the optimization tools offered by the Matlab software as the "fmincon.m" [27] function which allows the resolution of the nonlinear optimization problem with inequalities constraints, given by the procedure of computational of the robustness measure, presented in Theorem 4.5, and after some manipulations concerning the initialization task of the optimization algorithm under Matlab, we obtain the following optimal solution:

$$
P=\left[\begin{array}{cc}
0.0124 & 0 \\
0 & 0.1001
\end{array}\right], \quad \mu_{1}=0.0167, \quad \mu_{2}=0.0314, \quad \mu_{3}=0.0100
$$

and the system (4.18) is robustly stable for all uncertainty parameters $\varepsilon_{k j}$ such that $\left|\varepsilon_{k j}\right| \leq \eta_{2}^{\star}=$ 1.71 .

For testing the robustness bound gives above, we assume now that the model parameters are reversed compared to the nominal values, the simulation results, show that the perturbed system remains stable if the parameter uncertainties are not greater than the founded bound, which provide the validity of the presented approach. As compared to previously developed techniques like [21], the presented approach improve the robustness measure, which enlarges the uncertainties variation domain.

\section{Stabilizing Control Synthesis with Robustness Maximization}

In this section, we are interested the problem of nonlinear robust control of polynomial systems. In order to stabilize the considered nonlinear systems, a state feedback control law is built, based in recent and pertinent results about the polynomial systems stabilization using the LMI optimization tools (Linear Matrix Inequalities approach) and the robustness stability measure (4.16), proposed by Theorem 4.5 in Section 4 . An optimization algorithm allows to establish the following two conditions satisfied by the control law synthesis:

(i) the global stability of the nominal (without uncertainties) nonlinear polynomial system is ensured, and

(ii) the stability of the controlled perturbed system is ensured for a maximum bound of uncertainties affecting the system parameters.

\subsection{Nonlinear State Feedback Controller}

We consider now the uncertain polynomial system given by (3.1). Our purpose is to find a polynomial state feedback controller described by

$$
U=k(X)=\sum_{k=1}^{r} K_{k} X^{[k]}
$$


with $K_{k, k=1, \ldots, r}$ are constant gains matrices which stabilizes asymptotically and globally the equilibrium $(X=0)$ of the considered system (3.1) and to maximize the robustness measure. The controlled uncertain system is described by the state equation:

$$
\dot{X}=\sum_{k=1}^{r}\left(A_{k}+\Delta A_{k}\right) X^{[k]}
$$

where

$$
\begin{gathered}
A_{k}=F_{k}+B K_{k}, \\
\Delta A_{k}=\Delta F_{k}=\sum_{j=1}^{\gamma_{k}} \varepsilon_{k j} E_{k j} .
\end{gathered}
$$

\subsection{Robust Synthesis for Uncertain Polynomial Systems}

The robust stabilization technique presented in this work is specially related to a recent approach which deals with the global asymptotic stabilization of nonlinear polynomial systems within the framework of Linear Matrix Inequalities (LMIs) in the certain case, presented in [20]. The stabilization of nominal polynomial systems can be formulated as the following matrix inequality problem:

Find

(i) gain matrices $K_{i, i=1, \ldots, r}$,

(ii) a $(n \times n)$ matrix $P$, and

(iii) real parameters $\mu_{i, i=1, \ldots, \beta}$,

such that

$$
P>0,
$$

$$
\begin{aligned}
Q_{c} & =Q\left(P, \mu_{i, i=1, \ldots, \beta}, K_{k, k=1, \ldots, r}\right) \\
& =\tau^{T}\left(p(P) \mathcal{M}(f+B k)+\mathcal{M}(f+B k)^{T} P(P)\right) \tau+\Pi\left(\mu_{i, i=1, \ldots, \beta}\right)<0 .
\end{aligned}
$$

Let us note that this inequality (5.5) is a NLMI (Nonlinear Matrix Inequality) problem which can be turned into an LMI feasibility problem using specific transformations which are presented in [20].

Therefore, the proposed synthesis method combines the robust analysis result in the last section (Theorem 4.5) with the quadratic stabilization technique using the LMI approach in terms of a global optimization algorithm, presented above. Thus, our aim is to find a nonlinear state feedback controller (5.1) which stabilizes the closed loop system (5.2) and, at the same time, maximizes the bound on the perturbation parameters which the considered system can tolerate without going unstable. Then, our main result concerning the robust stabilization can be stated in the following theorem. 
Theorem 5.1. The controlled nonlinear uncertain polynomial system (5.2) is robustly asymptotically stable, if

$$
\eta_{c}=\max \left|\varepsilon_{k j}\right| \leq \eta_{c}^{\star}
$$

with

$$
\eta_{c}^{\star}=\max _{\left(P, \mu_{i}, K_{k}\right)}\left(\rho\left\{\sum_{k_{1}, j_{1}} \sum_{k_{2}, j_{2}}\left|\Omega_{k_{1} j_{1}} \Psi^{-1} \Omega_{k_{2} j_{2}} \Psi^{-1}+\Omega_{k_{2} j_{2}} \Psi^{-1} \Omega_{k_{1} j_{1}} \Psi^{-1}\right|+\sum_{k, j}\left|\left(\Omega_{k j} \Psi^{-1}\right)^{2}\right|\right\}\right)^{-1 / 2},
$$

where

$$
\begin{gathered}
\Psi=\mathcal{Q}_{c} \otimes I_{\vartheta}+I_{\vartheta} \otimes \mathcal{Q}_{c} \\
\mathcal{Q}_{c}=\mathcal{Q}\left(P, \mu_{i, i=1, \ldots, \beta}, K_{k, k=1, \ldots, r}\right)=\tau^{T}\left(p \mathcal{M}(f+B k)+\mathcal{M}(f+B k)^{T} p\right) \tau+\Pi\left(\mu_{i, i=1, \ldots, \beta}\right) .
\end{gathered}
$$

The above synthesis method can be seen as a nonlinear optimization procedure given by two step algorithm to found a global optimum of the problem (5.7). It should be mentioned that the resolution of the nonlinear optimization problem, presented in Theorem 5.1, is very sensitive to the initialization of the optimization algorithm. This difficulty is overcame simply when using the systematic results given by the LMI stabilization approach of nominal controlled polynomial systems, as a starting point for the nonlinear optimization described by the following algorithm.

Algorithm 5.2. One has the following steps:

Step 1 (Stabilization of the nominal system (Initialization)). Initialize the algorithm by determining a feasible solution using LMI technique of the nominal polynomial system: Solving the feasibility problem to find a symmetric positive matrix $P$, arbitrary parameters $\mu_{i, i=1, \ldots, \beta}$ and gain matrices $K_{k, k=1, \ldots, r}$ which globally stabilize the closed-loop nominal system. The obtained solution constitues the initialization parameters $P_{0}, \mu_{i_{0}}$ and $K_{k_{0}}$ of the next step. The second step allows the adjustment of the control gains.

Step 2 (Maximization of the robustness bound). Consider the matrices $K_{k, k=1, \ldots, r}, P$ and $\mu_{i, i=1, \ldots, \beta}$ obtained in Step 1 as initial values $\left(P_{0}, \mu_{i_{0}}\right.$ and $\left.K_{k_{0}}\right)$, and determine the optimal solution ensuring the stability of the controlled system (5.2) such that the following conditions are verified:

(i) $D=D^{T}$ is positive definite, and

(ii) $\mathcal{Q}_{c}\left(K_{k}, P, \mu_{i}\right)=\tau^{T}\left(D \mathcal{M}\left(A_{k, k=1, \ldots, r}\right)+\mathcal{M}\left(A_{k}, k=1, \ldots, r\right)^{T} D\right) \tau+\Pi\left(\mu_{i, i=1, \ldots, \beta}\right)$ is negative definite, and maximizing the robustness measure

$$
\eta_{c}^{\star}=\max _{\left(P, \mu_{i}, K_{k}\right)}\left(\rho\left\{\sum_{k_{1}, j_{1}} \sum_{k_{2}, j_{2}}\left|\Omega_{k_{1} j_{1}} \Psi^{-1} \Omega_{k_{2} j_{2}} \Psi^{-1}+\Omega_{k_{2} j_{2}} \Psi^{-1} \Omega_{k_{1} j_{1}} \Psi^{-1}\right|+\sum_{k, j}\left|\left(\Omega_{k j} \Psi^{-1}\right)^{2}\right|\right\}\right)^{-1 / 2},
$$


with

$$
\begin{gathered}
P>0, \\
\mathcal{Q}_{c}=\mathcal{Q}\left(P, A_{k, k=1, \ldots, r}, \mu_{i, i=1, \ldots, \beta}\right) \\
=\tau^{T}\left(p \mathcal{M}\left(A_{k, k=1, \ldots, r}\right)+\mathcal{M}\left(A_{k, k=1, \ldots, r}\right)^{T} D\right) \tau+\Pi\left(\mu_{i, i=1, \ldots, \beta}\right)<0 .
\end{gathered}
$$

As we indicated above, these two algorithm steps establish a nonlinear optimization problem under nonlinear constraints [27]. In the sequel, we give illustrative examples to show the effectiveness of the proposed algorithm.

\subsection{Numerical Example and Single Link Manipulator Application}

In this section, we present two simulation examples to demonstrate the validity of the proposed result such as numerical example and flexible manipulator with a single-link case study.

\subsubsection{Example 1: Numerical Example}

We consider the uncertain polynomial system described by the state equation (5.11), which the global asymptotic stabilization of the nominal part is treated in [20]:

$$
\dot{X}=\left(F_{1}+\Delta F_{1}\right) X+\left(F_{2}+\Delta F_{2}\right) X^{[2]}+\left(F_{3}+\Delta F_{3}\right) X^{[3]}+B U,
$$

where

$$
F_{1}=\left[\begin{array}{cc}
-1 & 1 \\
-1 & 1.5
\end{array}\right], \quad F_{2}=\left[\begin{array}{cccc}
1 & 1 & 0 & 0 \\
-1 & -0.5 & 0 & 0
\end{array}\right], \quad F_{3}=\left[\begin{array}{cccccccc}
-1 & 1 & 0 & -1 & 0 & 0 & 0 & 2 \\
-1 & -1 & 0 & 0.5 & 0 & 0 & 0 & -2
\end{array}\right]
$$

with $B=\left(\begin{array}{ll}0 & 1\end{array}\right)^{T}$, and

$$
\Delta F_{1}=\left[\begin{array}{cc}
\Delta F_{11} & 0 \\
0 & \Delta F_{14}
\end{array}\right], \quad \Delta F_{2}=\left[\begin{array}{cccc}
\Delta F_{21} & 0 & 0 & \Delta b_{24} \\
\Delta F_{25} & 0 & 0 & \Delta b_{28}
\end{array}\right], \quad \Delta F_{3}=\left[\begin{array}{cccccccc}
\Delta F_{31} & 0 & 0 & 0 & 0 & 0 & 0 & \Delta F_{38} \\
\Delta F_{39} & 0 & 0 & 0 & 0 & 0 & 0 & \Delta c_{46}
\end{array}\right] .
$$

First, according to Step 1, we are interested with the stabilization of the nominal part (i.e., global asymptotic stabilization of the origin equilibrium $(X=0))$ of the system $(5.11)$ using an LMI stabilization method developed in [20]. The obtained solution as initial condition of the next step is

$$
\begin{aligned}
\mu_{1_{0}} & =-0.0319, \\
\mu_{2_{0}} & =0.0246, \quad P_{0}=\left[\begin{array}{ll}
0.0246 & 0.0063 \\
0.0063 & 0.0071
\end{array}\right], \\
\mu_{3_{0}} & =0.1928,
\end{aligned}
$$


and the searched gain matrices which stabilizes the nominal closed-loop system:

$$
\begin{aligned}
& K_{1_{0}}=\left[\begin{array}{ll}
-9.8872 & -5.5518
\end{array}\right], \quad K_{2_{0}}=\left[\begin{array}{llll}
2.1050 & 0.8966 & 0 & 0
\end{array}\right],
\end{aligned}
$$

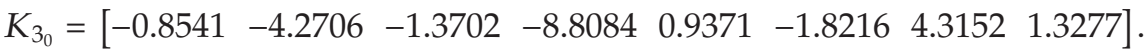

In the second step, solving the optimization problem [27] formulated by Theorem 5.1, we obtain:

$$
\begin{aligned}
& \mu_{1}=0.005 \\
& \mu_{2}=0.005 \\
& \mu_{3}=0.0707
\end{aligned} \quad P=\left[\begin{array}{cc}
0.0054 & 0.005 \\
0.005 & 0.0051
\end{array}\right] .
$$

The optimal control gain matrices are

$$
\begin{aligned}
& K_{1}=\left[\begin{array}{ll}
1.7811 & -2.5004
\end{array}\right], \quad K_{2}=\left[\begin{array}{llll}
-1.2187 & 0.0500 & -0.7886 & 0.0227
\end{array}\right], \\
& K_{3}=\left[\begin{array}{llllllll}
-1.2343 & -6.9917 & -4.1103 & 2.4763 & 12.0761 & -4.5891 & 1.5424 & 0.3126
\end{array}\right] \text {. }
\end{aligned}
$$

The stability of the uncertain system is guaranteed for all uncertainty parameters $\varepsilon_{k j}$ such that $\max \left(\varepsilon_{k j}\right)=\eta_{c} \leq \eta_{c}^{\star}=2.3542$.

The system controlled with the obtained polynomial control law was simulated for a perturbation of $100 \%$ on the state variable $x_{1}$ and parametric perturbation corresponding to values of $\varepsilon_{k j}=2.3542$. Figure 1 presents the state trajectories of the both nominal and perturbed polynomial system with the proposed nonlinear polynomial controllers. The simulation depicted in Figure 2 presents the evolution of the performed feedback stabilizing polynomial controller.

\subsubsection{Example 2: Single-Link Manipulator Application}

To study the validity of the proposed approach in control of robot manipulator application, we consider a single-link manipulator with flexible joints and negligible damping represented by Figure 3 [28].

We model the dynamics of this system ignoring damping terms as

$$
(I+J) \ddot{q}+B \dot{q}+M g l \sin (q)=u
$$

which can be expressed as

$$
\begin{gathered}
I \ddot{q}_{1}+M g l \sin \left(q_{1}\right)+k\left(q_{1}-q_{2}\right)=0, \\
J \ddot{q}_{2}-k\left(q_{1}-q_{2}\right)-u=0 .
\end{gathered}
$$

The parameters used in these equations are defined as follows: $I$ is the link inertia, $J$ represents the rotor inertia, $q$ is the vector of link positions, $M g l$ nominal load, $B$ rotor friction, $k$ denotes the joint stiffness and $u$ represents the input torque. 

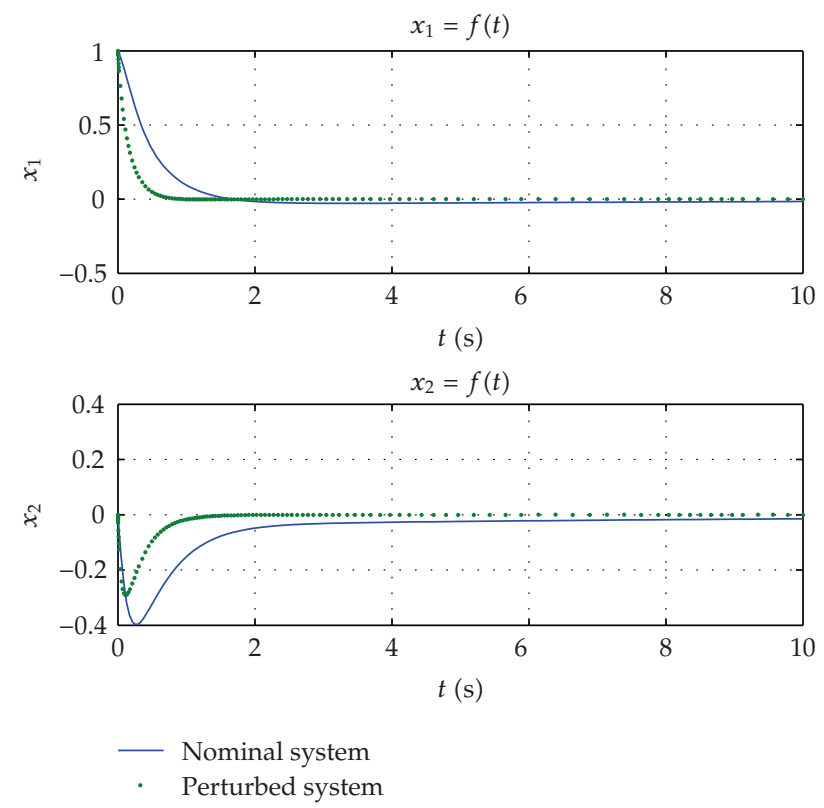

Figure 1: Behavior of the state variables of both nominal and perturbed system.

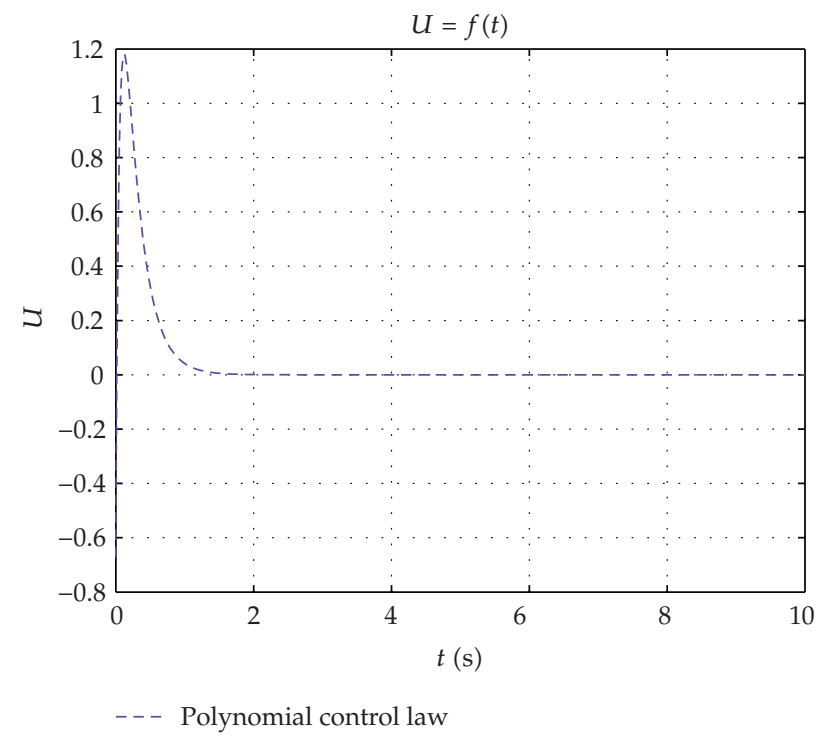

Figure 2: Simulation curve of the proposed feedback controller.

The considered system can be described by a fourth-order model of the form:

$$
\dot{X}=f(X)+B u,
$$




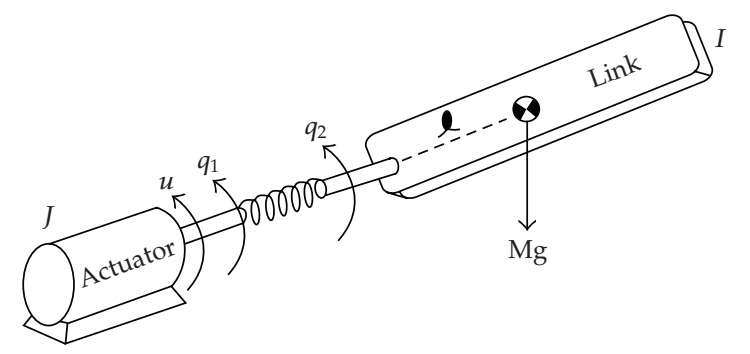

Figure 3: Model of single-link manipulator with flexible joint.

where $X$ is the state vector defined by

$$
\begin{aligned}
& X=\left[\begin{array}{llll}
q_{1} & \dot{q}_{1} & q_{2} & \dot{q}_{2}
\end{array}\right]^{T}=\left[\begin{array}{llll}
x_{1} & x_{2} & x_{3} & x_{4}
\end{array}\right]^{T}, \\
& f(X)=\left[\begin{array}{c}
\dot{q}_{1} \\
-a \sin \left(q_{1}\right)-b\left(q_{1}-q_{2}\right) \\
\dot{q}_{2} \\
c\left(q_{1}-q_{2}\right)
\end{array}\right],
\end{aligned}
$$

and $B=\left[\begin{array}{llll}0 & 0 & 0 & d\end{array}\right]^{T}$ with

$$
a=I^{-1} M g l, \quad b=I^{-1} k, \quad c=J^{-1} k, \quad d=J^{-1} .
$$

The nonlinear system (5.20) can be developed into polynomial form by a Taylor series expansions, then we have

$$
\dot{X}=\left(F_{1}+\Delta F_{1}\right) X+\left(F_{2}+\Delta F_{2}\right) X^{[2]}+\left(F_{3}+\Delta F_{3}\right) X^{[3]}+B U,
$$

where

$$
F_{1}=\left[\begin{array}{cccc}
0 & 1 & 0 & 0 \\
-(a+b) & 0 & b & 0 \\
0 & 0 & 0 & 1 \\
c & 0 & -c & 0
\end{array}\right], \quad F_{2}=0_{4 \times 16}
$$

$F_{3}(1,1)=a / 6 ; F_{3}(i, j)=0$ for all $i, j(i=1, \ldots, 4 ; j=1, \ldots, 64)$, and

$$
\Delta F_{1}=\left[\begin{array}{cccc}
0 & 0 & 0 & 0 \\
\Delta F_{11} & 0 & \Delta F_{12} & 0 \\
0 & 0 & 0 & 0 \\
\Delta F_{13} & 0 & \Delta F_{14} & 0
\end{array}\right], \quad \Delta F_{2}=0_{4 \times 16}
$$

$\Delta F_{3}(1,1)=\Delta F_{31} ; \Delta F_{3}(i, j)=0$ for all $i, j \quad(i=1, \ldots, 4 ; j=1, \ldots, 64)$. 


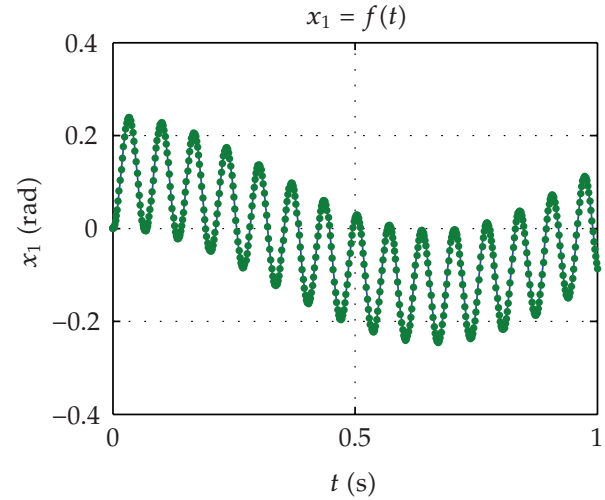

(a)

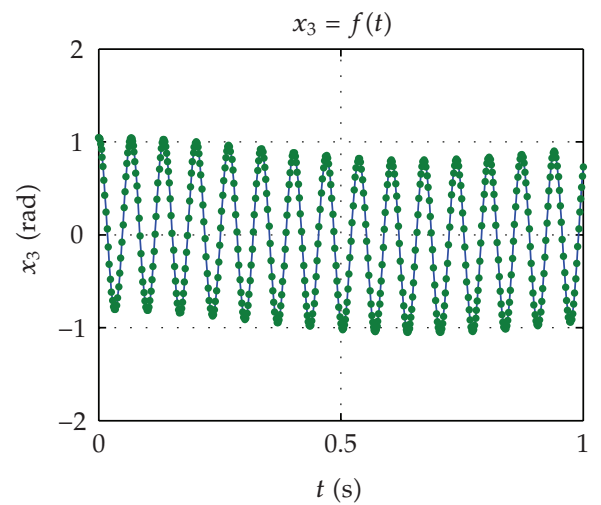

(c)

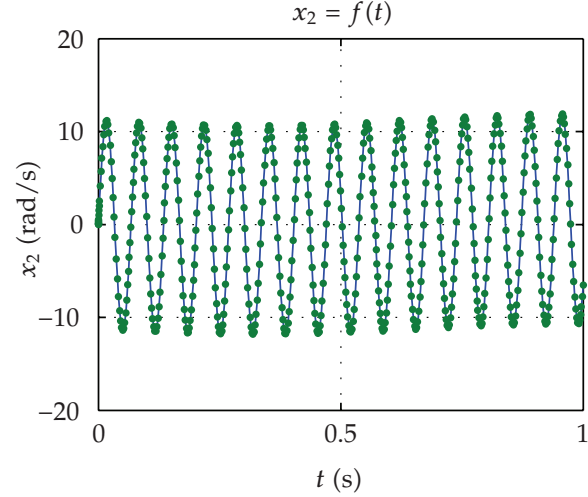

(b)

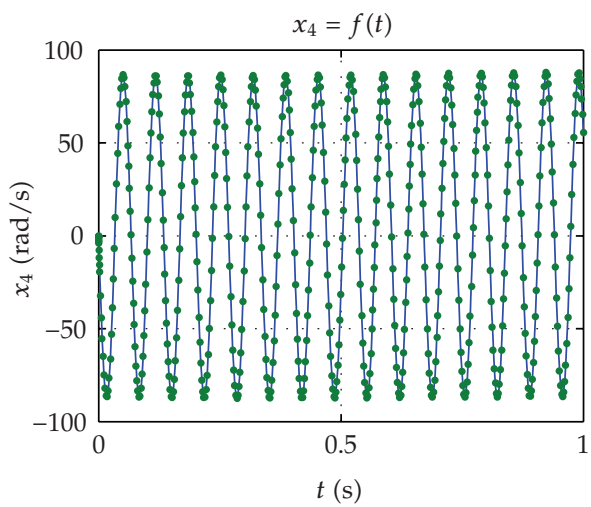

(d)

Figure 4: Evolution of state variables without controller for both real model (-) and polynomial model $(\bullet \bullet)$.

Table 1: Nominal values of the system parameters.

\begin{tabular}{lc}
\hline Link inertia, $I$ & $0.031 \mathrm{Kg} \cdot \mathrm{m}^{2}$ \\
Rotor inertia, $J$ & $0.004 \mathrm{Kg} \cdot \mathrm{m}^{2}$ \\
Rotor friction, $B$ & $0.007 \mathrm{~N} . \mathrm{m} . \mathrm{sec} / \mathrm{rad}$ \\
Nominal load, $M g l$ & $0.8 \mathrm{~N} \cdot \mathrm{m}$ \\
Joint stiffness, $k$ & $31.0 \mathrm{~N} \cdot \mathrm{m} / \mathrm{rad}$ \\
\hline
\end{tabular}

For simulation, the single-link manipulator parameters are considered in Table 1 [29]. Thus, according to the values of the considered system parameters indicated in Table 1 and an initial condition is assumed to be

$$
\left[\begin{array}{llll}
q_{1}(0) & \dot{q}_{1}(0) & q_{2}(0) & \dot{q}_{2}(0)
\end{array}\right]^{T}=\left[\begin{array}{llll}
0 & 0 & \frac{\pi}{3} & 0
\end{array}\right]^{T} .
$$

The evolution of the state variables of the single-link manipulator without controller is shown in Figure 4. It appears in the simulations that the polynomial approach is a more objective of the real system, since, we can't distinguish the real system behavior and the polynomial one. 


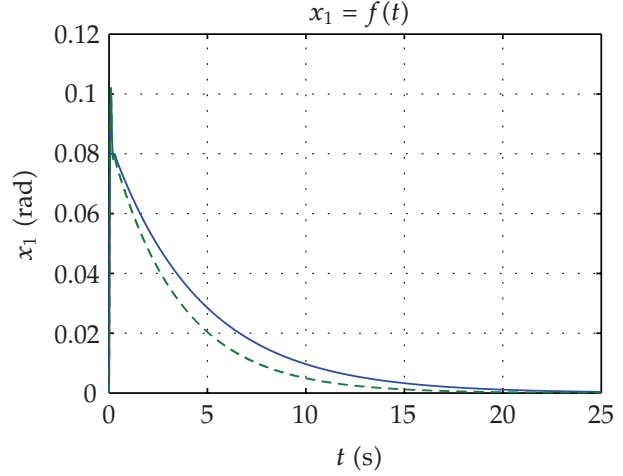

(a)

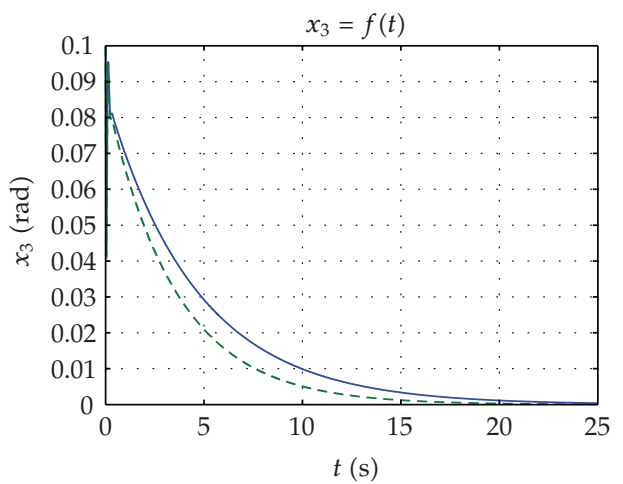

- Nominal system

- - - Perturbed system

(c)

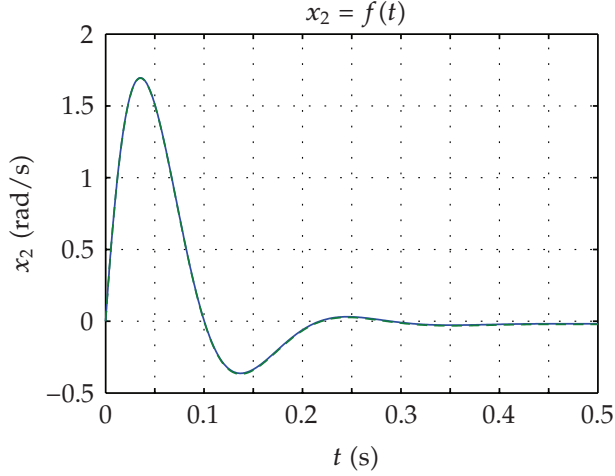

(b)

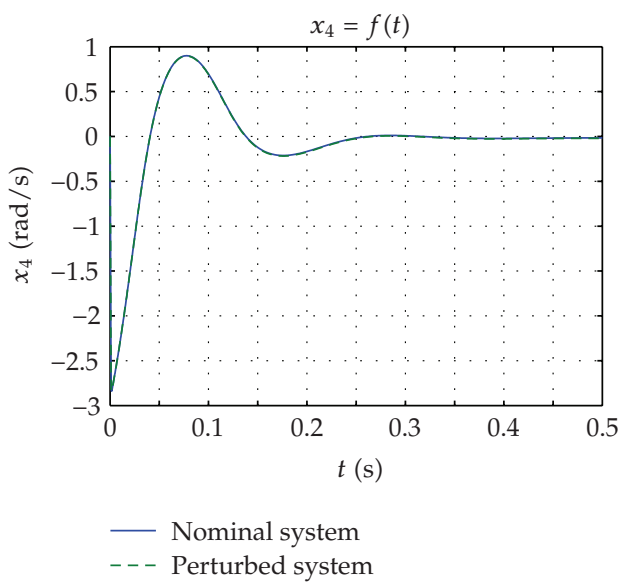

(d)

Figure 5: Closed-loop system response using the polynomial controller.

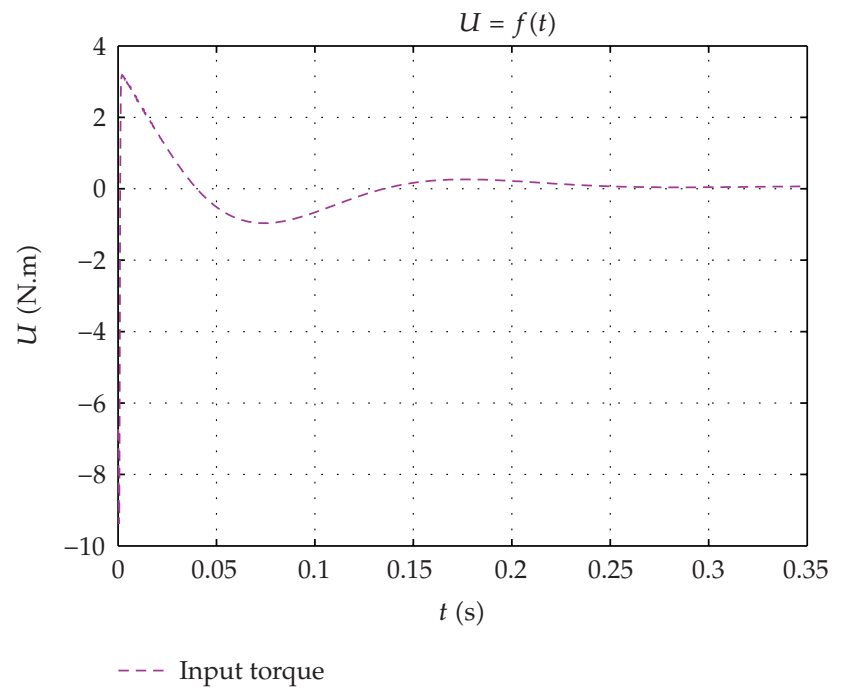

Figure 6: Simulation of the stabilizing polynomial control (5.28). 
In order to investigate the performance of the proposed synthesis method, applying the optimization algorithm presented in the section above, we obtain the following optimal solution:

$$
P=\left[\begin{array}{llll}
0.0005 & 0.0172 & 0.0002 & 0.0007 \\
0.0172 & 0.5958 & 0.0083 & 0.9066 \\
0.0002 & 0.0083 & 0.0005 & 0.0189 \\
0.0007 & 0.3066 & 0.0189 & 0.1582
\end{array}\right]
$$

The obtained state feedback control law

$$
U=K_{1} X+K_{2} X^{[2]}+K_{3} X^{[3]}
$$

is builded with the following optimal gains matrices:

$$
\begin{aligned}
& K_{1}=\left[\begin{array}{llll}
71.345 & -0.3219 & -69.4698 & -2.9487
\end{array}\right], \\
& \left.K_{2}=\begin{array}{llllllll}
-0.0033 & -0.0034 & -0.0678 & -0.00819 & -0.0123 & -0.4854 & -0.0086 & -0.0065 \\
-0.0345 & -0.0059 & -0.0295 & -0.0003 & -0.0045 & -0.0023 & -0.089 & -0.0196
\end{array}\right] \text {, } \\
& {\left[\begin{array}{llllllll}
73.1389 & -0.0039 & -0.0079 & -0.0057 & -0.9574 & -0.0049 & -0.0052 & -0.0084
\end{array}\right.} \\
& \begin{array}{lllllllll}
-68.1638 & -0.0052 & -0.0027 & -0.0026 & -2.3459 & -0.0023 & -0.0025 & -0.0035
\end{array} \\
& \begin{array}{lllllllll}
-0.005278 & 71.4631 & -0.0023 & -0.0034 & -0.0010 & -0.1539 & -0.0015 & -0.0034
\end{array} \\
& K_{3}=\begin{array}{lllllllll}
-0.0026 & -69.9302 & -0.0012 & -0.0015 & -0.0014 & -2.2098 & -0.0034 & -0.0034 \\
-0.0024 & -0.0045 & 69.4388 & -0.0036 & -0.0035 & -0.0201 & -0.1123 & -0.0045
\end{array} \\
& \begin{array}{lllllllll}
-0.0088 & -0.0049 & -73.3322 & -0.0069 & -0.0062 & -0.0063 & -2.2398 & -0.0057
\end{array} \\
& \begin{array}{lllllllll}
-0.0057 & -0.0015 & -0.0045 & 65.5724 & -0.0035 & -0.0057 & -0.0047 & -0.5739
\end{array} \\
& \begin{array}{llllllll}
-0.0046 & -0.0013 & -0.0013 & -64.5544 & -0.0024 & -0.0086 & -0.0024 & -2.3040] \text {. }
\end{array}
\end{aligned}
$$

The closed-loop system responses using the obtained polynomial controller are depicted in Figure 5.

As it can be seen, the nonlinear state feedback controller (5.28) stabilizes the considered system. Moreover, the proposed robust controller, shown in Figure 6, can rapidly damp the oscillations of the manipulator and greatly enhance transient stability for all uncertainty parameters $\varepsilon_{k j}=\Delta F_{k j} \leq 1.0581$.

\section{Conclusion}

In this paper, we have investigated the problems of robust stability analysis and robust stabilization for a class of uncertain nonlinear systems with structured uncertainties. New developments on the analysis and control of nonlinear polynomial systems have been 
presented. The combination of a polynomial system stability criterion with an improved robustness measure of uncertain linear systems has allowed the formulation of a new criterion for robustness bound estimation of the studied uncertain polynomial systems. When compared with the previous results, this developed criterion led to a wider robustness measure. It has been shown from the simulation results that the proposed robust control scheme is efficient and permits the rapid stabilization of the nominal system and the maximization of the uncertainties bound. A future work will be to establish the robust stability and stabilization of uncertain polynomial systems with both structured and unstructured uncertainties. Moreover, the problem of feedback $H_{\infty}$ control for the studied class of nonlinear uncertain systems presents another interesting topic for future research.

\section{Appendix}

Proof Elements of Theorem 4.3 and Relative Notations

For the complete proof of Theorem 4.3, we can refer to $[15,16,19]$. We limit here to remind the fundamental principles, we consider the following quadratic lyapunov function $\mho(X)=$ $X^{T} P X$, then we can show that:

$$
\frac{d U(X)}{d t}=-\tilde{x}^{\mathrm{T}} Q \tilde{x}
$$

where $\tilde{x}$ is the $\vartheta$-vector defined by

$$
\tilde{X}=\left[\begin{array}{llll}
\tilde{X}^{T} & \tilde{X}^{[2]^{T}} & \cdots & \tilde{X}^{[s]^{T}}
\end{array}\right]^{T}
$$

and $Q$ is the symmetric matrix defined in (4.7), where

$$
\begin{gathered}
P=\operatorname{diag}\left(P, P \otimes I_{n}, \ldots, P \otimes I_{n^{s-1}}\right), \\
X=\tau \tilde{x},
\end{gathered}
$$

and $\tau$ is defined by:

$$
\tau=\operatorname{diag}\left(T_{1}, T_{2}, \ldots, T_{s}\right)
$$

with $\mathcal{M}(f)$ is the matrix of the system parameters, expressed by (2.8).

The matrix $\Pi\left(\mu_{i, i=1, \ldots, \beta}\right)$ is defined as

$$
\Pi\left(\mu_{i, i=1, \ldots, \beta}\right)=\sum_{i=1}^{\beta} \mu_{i} \operatorname{mat}_{(\eta, \eta)}\left(C_{i}\right)
$$

where the columns $C_{i, i=1, \ldots, \beta}$ and the coefficients $\mu_{i}$ and $\beta$ are detailed in [15]. 


\section{References}

[1] S. Jannesari, "Stability analysis and stabilization of a class of nonlinear systems based on stability radii," in International Conference on Control, Control, vol. 1, pp. 641-645, Swansea, UK, 1998.

[2] J. Zhijian and W. Long, "Robust stability and stabilization of a class of nonlinearswitched systems," in Proceedings of the 25th IASTED International Conference on Modelling, Indentification, and Control, pp. 37-42, Lanzarote, Spain, 2006.

[3] M. S. Mahmoud and N. B. Almutairi, "Robust stability and stabilization methods for a class of nonlinear discrete-time delay systems," Applied Mathematics and Computation, vol. 215, no. 12, pp. 4280-4292, 2010.

[4] Y. Wang, L. Xie, and C. E. de Souza, "Robust control of a class of uncertain nonlinear systems," Systems \& Control Letters, vol. 19, no. 2, pp. 139-149, 1992.

[5] D. F. Coutinho, M. Fu, and A. Trofino, "Robust analysis and control for a class of uncertain nonlinear discrete-time systems," Systems E Control Letters, vol. 53, no. 5, pp. 377-393, 2004.

[6] J. Yoneyama, "Robust stability and stabilization for uncertain Takagi-Sugeno fuzzy time-delay systems," Fuzzy Sets and Systems, vol. 158, no. 2, pp. 115-134, 2007.

[7] M. De la Sen, "Robust stability analysis and dynamic gain-scheduled controller design for point timedelay systems with parametrical uncertainties," Communications in Nonlinear Science and Numerical Simulation, vol. 13, no. 6, pp. 1131-1156, 2008.

[8] V. F. Montagner, R. C. L. F. Oliveira, T. R. Calliero, R. A. Borges, P. L. D. Peres, and C. Prieur, "Robust absolute stability and nonlinear state feedback stabilization based on polynomial Lur'e functions," Nonlinear Analysis: Theory, Methods \& Applications, vol. 70, no. 5, pp. 1803-1812, 2009.

[9] R. V. Patel and M. Toda, "Quantitative measures of robustness for multivariable systems," in Proceedings of Joint Automatic Control Conference, San Francisco, Calif, USA, 1980.

[10] R. K. Yedavalli, "Flight control application of new stability robustness bound for linear uncertain systems," Journal of Guidance, Control, and Dynamics, vol. 16, no. 6, pp. 1032-1037, 1993.

[11] J. H. Kim, "Robust stability of linear systems with delayed perturbations," IEEE Transactions on Automatic Control, vol. 41, no. 12, pp. 1820-1822, 1996.

[12] J. D. Gardiner, "Computation of stability robustness bounds for state-space models with structured uncertainty," IEEE Transactions on Automatic Control, vol. 42, no. 2, pp. 253-256, 1997.

[13] X. Li and C. E. De Souza, "Criteria for robust stability and stabilization of uncertain linear systems with state delay," Automatica, vol. 33, no. 9, pp. 1657-1662, 1997.

[14] F. Rotella and G. Dauphin-Tanguy, "Non-linear systems: identification and optimal control," International Journal of Control, vol. 48, no. 2, pp. 535-544, 1988.

[15] N. Benhadj Braiek, F. Rotella, and M. Benrejeb, "Algebraic criteria for global stability analysis of nonlinear systems," International Journal of Systems Analysis Modelling and Simulation, vol. 17, pp. 221227, 1995.

[16] N. Benhadj Braiek, "On the global stability of nonlinear polynomial systems," in Proceedings of the 35th IEEE Conference on Decision and Control (CDC'96), Kobe, Japan, December 1996.

[17] H. Bouzaouache and N. Benhadj Braiek, "On the stability analysis of nonlinear systems using polynomial Lyapunov functions," Mathematics and Computers in Simulation, vol. 76, no. 5-6, pp. 316329, 2008.

[18] J. W. Brewer, "Kronecker products and matrix calculus in system theory," IEEE Transactions on Circuits and Systems, vol. 25, no. 9, pp. 772-781, 1978.

[19] R. Mtar, M. M. Belhaouane, H. Belkhiria Ayadi, and N. Benhadj Braiek, "An LMI criterion for the global stability analysis of nonlinear polynomial systems," Nonlinear Dynamics and Systems Theory, vol. 9, no. 2, pp. 171-183, 2009.

[20] M. M. Belhaouane, R. Mtar, H. Belkhiria Ayadi, and N. Benhadj Braiek, "An LMI technique for the global stabilization of nonlinear polynomial systems," International Journal of Computers, Communications and Control, vol. 4, no. 4, pp. 335-348, 2009.

[21] N. Benhadj Braiek and H. Belkhiria, "Robustness stability measure for a class of nonlinear uncertain systems," in Proceedings of IEEE International Conference on Systems, Man, and Cybernetics (SMC '99), vol. 1, pp. 28-32, Tokyo, Japan, October 1999.

[22] H. Belkhiria Ayadi and N. Benhadj Braiek, "Robust control of nonlinear polynomial systems," in Proceedings of IEEE International Conference on Systems, Man, and Cybernetics (SMC '99), vol. 4, pp. 1-5, Yasmine Hammamet, Tunisia, 2002.

[23] N. Benhadj Braiek and F. Rotella, "Logid: a nonlinear systems identification software," in Modelling and Simulation of Systems, pp. 211-218, Scientific Publishing, 1990. 
[24] R. K. Yedavalli, "Improved measures of stability robustness for linear state space models," IEEE Transactions on Automatic Control, vol. 30, no. 6, pp. 577-579, 1985.

[25] R. K. Yedavalli and Z. Liang, "Reduced conservatism in stability robustness bounds by state transformation," IEEE Transactions on Automatic Control, vol. 31, no. 9, pp. 863-866, 1986.

[26] H. K. Khalil, Nonlinear Systems, Prentice-Hall, Upper Saddle River, NJ, USA, 3rd edition, 2000.

[27] T. Coleman, M. Ann Branch, and A. Grace, "Optimization toolbox for use withMatlab," User's guide, version 2, third printing revised for version 2 (Matlabrelease 11), January 1999.

[28] M. W. Spong, "Adaptive control of flexible joint manipulators," Systems E Control Letters, vol. 13, no. 1, pp. 15-21, 1989.

[29] F. Ghorbel, J. Y. Hung, and M. W. Spong, "Adaptative control of flexible joint manipulators," in Proceedings of IEEE International Conference on Robotics and Automation, vol. 2, pp. 1188-1193, Scottsadale, Ariz, USA, May 1989. 


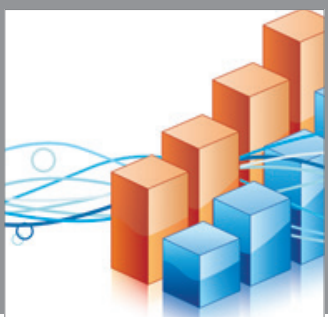

Advances in

Operations Research

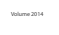

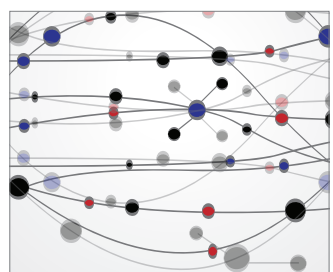

\section{The Scientific} World Journal
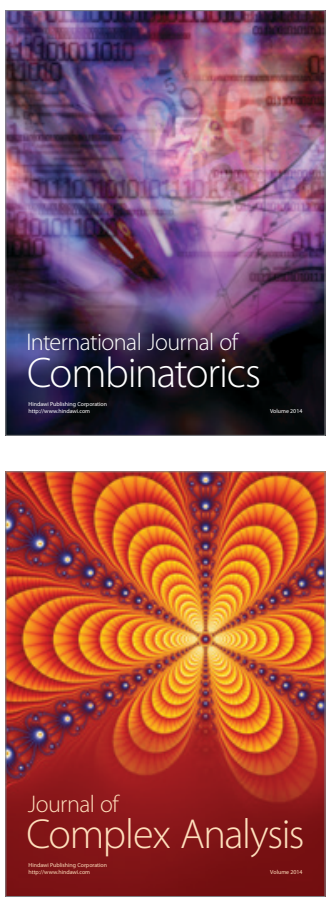

International Journal of

Mathematics and

Mathematical

Sciences
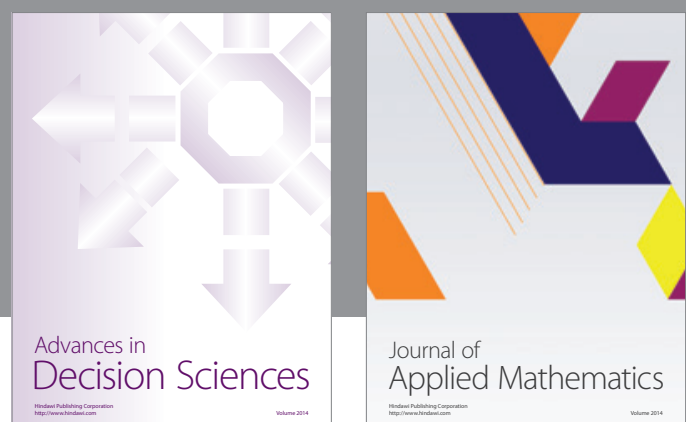

Journal of

Applied Mathematics
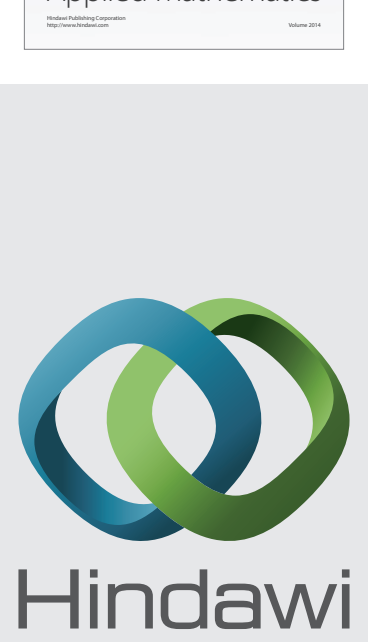

Submit your manuscripts at http://www.hindawi.com
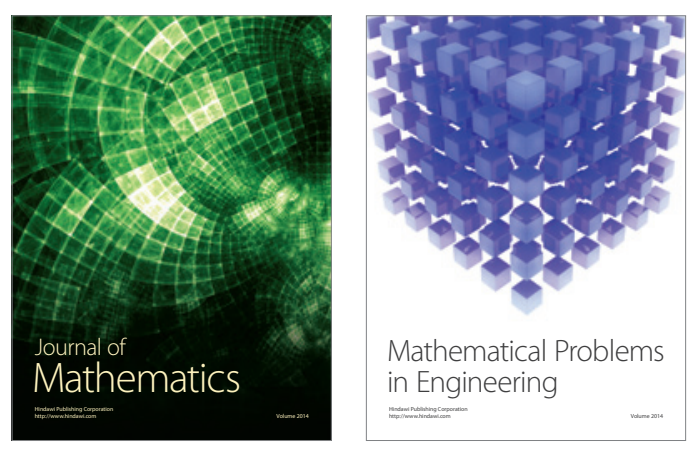

Mathematical Problems in Engineering
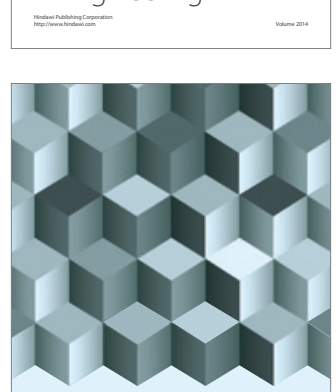

Journal of

Function Spaces
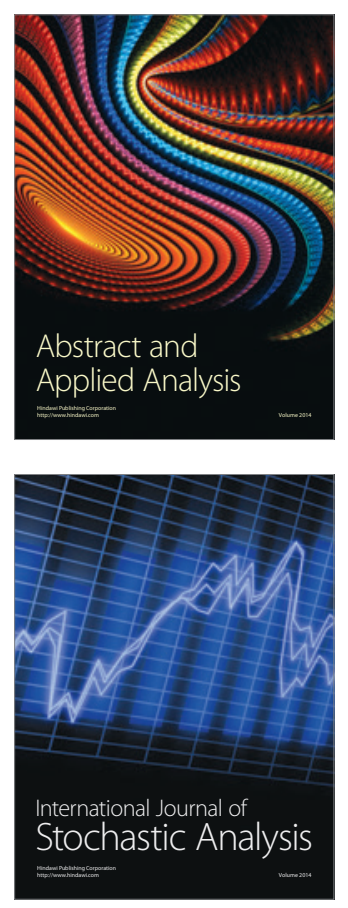

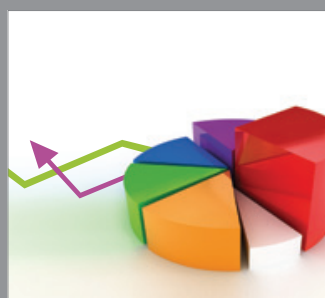

ournal of

Probability and Statistics

Promensencen
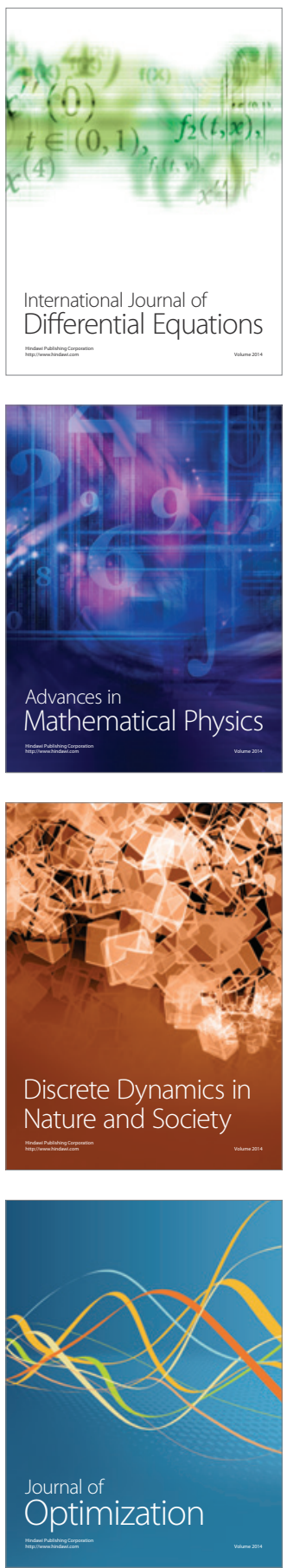